\title{
Synchrotron Diffraction topography in Studying of the Defect Structure in Crystals Grown by the Czochralski Method
}

\author{
W. Wierzchowski ${ }^{a *}$, K. Wieteska ${ }^{b}$, A. Malinowska $^{a}$, E. WierzbickA $^{a}$, \\ M. LeFEld-SOSNOWskA ${ }^{c}$, M. ŚWIRKOWICZ ${ }^{a}$, T. EukASIEWICZ ${ }^{a}$, A. PAJĄCZKOWskA $^{a}$ \\ AND C. PAULMANN ${ }^{d}$ \\ ${ }^{a}$ Institute of Electronic Materials Technology, Wólczyńska 133, 01-919 Warsaw, Poland \\ ${ }^{b}$ National Centre for Nuclear Research, A. Sołtana 7, 05-400 Otwock-Świerk, Poland \\ ${ }^{c}$ Institute of Experimental Physics, Faculty of Physics, University of Warsaw, Hoża 69, 00-681 Warsaw, Poland \\ ${ }^{d}$ HASYLAB at DESY, Notkestr. 85, D-22607 Hamburg, Germany

\begin{abstract}
The synchrotron diffraction topography had been widely used for investigation of the structural defects in crystals grown by the Czochralski method. Similarly as conventional diffraction topography, the synchrotron topography consists in recording with high spatial resolution of the beam formed by the Bragg reflection from the crystal. The advantages of synchrotron sources come from the possibilities of using the wavelength from a wide spectral range, improved high spatial resolution and collimation of the beam as well as from shortening the time necessary for the investigation. The synchrotron diffraction topography includes experimentally simpler white beam topography and more complicated monochromatic beam (multicrystal) topography, where the beam is formed by monochromators. In the case of Czochralski-grown crystals the synchrotron diffraction topography can be used for studying of the individual dislocations and their complexes such as glide bands or sub-grain boundaries, individual blocks, twinning, the domain structure and various segregation effects negatively affecting crystal properties. In addition, the topographical investigation can provide information concerning the reasons for the generation of defects, useful in the improving of the technology. In the present paper the possibilities of the synchrotron diffraction topography are discussed on the basis of several investigations of the Czochralski-grown oxide and semiconductor crystals, performed by the authors at HASYLAB. The majority of the results concern the oxide crystals grown at the Institute of Electronic Materials Technology, in particular garnets, orthovanadates, mixed calcium barium and strontium niobates as well as praseodymium lanthanum aluminates.
\end{abstract}

DOI: 10.12693 /APhysPolA.124.350

PACS: 61.72.Ff

\section{Introduction}

The basic principle of the X-ray diffraction topography consists in the recording of the beam Bragg reflected from the crystal with high spatial resolution and the revealing of the defects thanks to the local changes of the reflected beam intensity and beam direction changes in white beam topography. Historically the first attempts of the topographic investigations started in thirties years of 20th century by Berg [1] followed later by Barrett [2], Ramachandran [3], Guinier and Tennevin [4], Bond and Andrus [5] and Schulz [6]. In the first period the most important difficulty was inadequate methods of surface preparation later solved by various methods of mechanically-chemical polishing.

The fast development of the method was connected with the development of the semiconductor technology and other application of crystalline materials e.g. in laser technology. The diffraction topography became very popular in view of its non-destructive character and the

\footnotetext{
* corresponding author; e-mail:

Wojciech.Wierzchowski@itme.edu.pl
}

possibilities of the interpretation of the revealed defects in terms of crystallography and theory of elasticity.

The conventional X-ray diffraction topography is based on the two main concepts. We owe the first one to Lang $[7,8]$ who used the narrow beam collimated by slits restricting the spectral range to a single $K_{\alpha 1}$ line. The possibilities of obtaining large area topographs were achieved by his idea of simultaneous translation of the crystal coupled with film in respect of the beam. The derivative method is the section topography, where the exposures are taken without translation. The important detail in the section topography is the use of a narrow beam limited with the $5-30 \mu \mathrm{m}$ wide slit. The methods proposed by Lang had been widely used for characterization of Czochralski-grown crystals as it was discussed in a review book by Tanner [9].

Another important method of X-ray diffraction topography is assigned to Bonse and Kappler [10, 11] who firstly published the images of dislocations and theoretical explanation of contrast, but is an extension of Bond and Andrus idea [5]. The method is often called double-crystal topography and consists in taking the topographs using monochromator, possibly with the same or very close interplanar distances of the reflecting planes as those in the investigated crystal. Some important 
improvement of the method consists in applying of the monochromator with suitable asymmetry [5, 12, 13]. The double-crystal method is experimentally more difficult and less popular than the methods proposed by Lang.

As in the case of other X-ray methods the significant development of X-ray diffraction topography was caused by wide availability of the synchrotron radiation offering some important advantages, as the possibilities of choosing the wavelength, achieving of high spatial resolution, more effective collimation of the beam and shortening of the time necessary for the investigation.

Topographic experiments with the use of synchrotron radiation were firstly described by Tuomi [14] and were followed by Hart et al. [15], Tanner et al. [16, 17], Hart et al. [18], Petroff and Sauvage [19], Sauvage [20] and other authors [21-23]. A review of papers concerning the applications of synchrotron topography until was given by Bowen and Tanner [24], and later by Authier in his book about the dynamical theory of X-ray diffraction [25]. In synchrotron topography one can distinguish single crystal topography called also white beam topography [26, 27], and multi-crystal topography also called the monochromatic beam topography, where the beam is formed by reflection from one [28] or more crystals $[19,20]$. Apart from white beam projection topography one can use white beam section and even pin-hole topography using either a very narrow or pencil-like beam, respectively.

An interesting possibility is the "stroboscopic topography" exploring the fact that synchrotron beam is formed by a series of accurately time-spaced bunches. It is then possible to reveal the different stages of the harmonic oscillators in the crystals if they are synchronized with the frequency of bunches in the synchrotron beam [21-23]. Such experiments were in particular performed in the Czochralski-grown lithium niobate crystals [23].

A systematic development of electronic imaging devices made possible to achieve the spatial resolution of single $\mu \mathrm{m}$ with the use of CCD camera (the one with $3 \mu \mathrm{m}$ resolution is used at ANKA TOPO-station) but still the best results are available with high resolution photographic materials, especially holographic films and nuclear emulsions.

Similarly as in the case of conventional X-ray topography there are several methods enabling the identification of defects, especially dislocations, stacking faults and regularly shaped inclusions. These methods include the extinction analysis in various crystallographically equivalent reflections and the simulation of topographic contrasts $[9,24,25]$. These methods are even more effective in case of synchrotron than in conventional topography thanks to the possibility of better resolution and better collimation and using more suitable wavelength forming the image [24, 25]. The numerical simulation of contrast can now be realized with fast modern personal computers practically in case of all topographic methods, including the simulation of traverse topographs requiring the time even shorter than $1 \mathrm{~h}$.
The synchrotron topographic investigations of the Czochralski grown crystals were published by Baruchel et al. [29], Malinowska et al. [30], Kasper et al. [31], Yao et al. [32], Muehlberg et al. [33], Prokhorov et al. [34], Yoneda et al. [35] and by Dudley and Yao [36, 37]. Present paper will illustrate the possibilities of the synchrotron X-ray topography in the Czochralski grown crystals and some more detailed experimental problem by several results obtained during several years at HASYLAB, which were published elsewhere [38-54].

\section{Experimental realization of synchrotron diffraction topography}

The popular sources of the synchrotron radiation, used also in the case of presently described topographic experiments at DORIS III are bending magnets. The high energy of the positron beam equal to $4.5 \mathrm{GeV}$ allowed obtaining the photon energy in the range 5 to $35 \mathrm{keV}$, which is satisfactory for realization of the most of the topographic investigation. The present investigations of the Czochralski grown crystals were realized at the two experimental stations - the white beam station F1 and the monochromatic beam station E2.

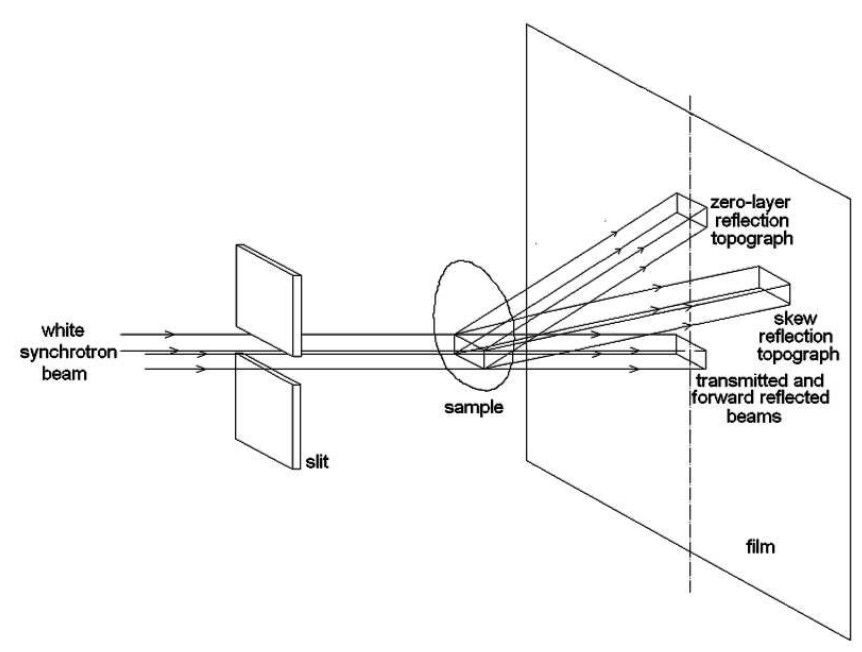

Fig. 1. The scheme of the synchrotron white beam topography in transmission case.

In the case of the white beam topography the beam coming directly from the synchrotron through the slit system is used as it is schematically shown in Fig. 1. The beam limited by a system of vertical and horizontal slits is entering the crystals to obtain a kind of Laue pattern either in the the Laue case (transmission) or Bragg case (reflection). Thanks to small apparent diameters of the synchrotron source, here approximately $0.45 \mathrm{~mm}$ in vertically and $1.5 \mathrm{~mm}$ horizontally and large $18 \mathrm{~m}$ distance of the experimental station from the bending magnet, each Laue spot is a high resolution topograph revealing the defects in the investigated crystal. The spatial resolutions $w_{\mathrm{h}, \mathrm{v}}$ may be evaluated from the simple formula 


$$
w_{\mathrm{h}, \mathrm{v}}=\frac{d_{\mathrm{fk}}}{d_{\mathrm{sk}}} f_{\mathrm{h}, \mathrm{v}},
$$

where $d_{\mathrm{fk}}$ is film-to-crystal distance, $d_{\mathrm{sk}}$ is the distance of the crystal from the bending magnet or insertion device and $f_{\mathrm{h}, \mathrm{v}}$ are apparent diameters of the radiation source. Differently than in the conventional Lang topography the resolution in the direction parallel to the diffraction plane is determined mainly by the divergence of the beam and the same formula can be applied for evaluation both of vertical and horizontal resolution. At typical distances of film-to-crystal of $5 \mathrm{~cm}$ the horizontal spatial resolution is better than $4 \mu \mathrm{m}$, while the vertical is better than $1 \mu \mathrm{m}$. The apparent diameters of radiation source are significantly smaller in case of III generation synchrotron sources e.g. $0.2 \mathrm{~mm} \times 0.4 \mathrm{~mm}$ in the case of bending magnets at ESRF.

A significant limitation of the beam is necessary for several reasons, in particular to decrease the scattering of the high flux beam by air and various parts of the experimental equipment, to reduce the heat load effect and to avoid the overlapping of the spots. In view of that, the beam size did not exceed $3 \times 3 \mathrm{~mm}^{2}$. The equivalent horizontally elongated beam of $0.8 \times 7 \mathrm{~mm}^{2}$ was more convenient. The heat-load effect can be dangerous in case of some highly absorbing or temperature unstable crystals, particularly limiting the possibility of using of low sensitive photographic material with high resolution. In order to study larger samples, a series of topographs after suitable translations sample should be taken. In case of the section topography the width of the slit was closed to $5 \mu \mathrm{m}$. The present investigations were performed both in the reflection and transmission case with the projection and section topographs.

The white beam synchrotron topography is equivalent to the Shultz [6] and differs from the conventional Lang method, especially the case when the crystals contain several misoriented parts. Here the synchrotron white beam topographs provide the images of all these parts, however, the beams forming the images are differently directed, according to the rule that the angle of incidence on the reflecting plane is equal to the angle of reflection. The white beam synchrotron topography is only sensitive to the inclination component of the strain field and not sensitive to lattice parameter changes. The white-beam projection and section of topographic images of almost perfect crystals containing the individual defects are otherwise very similar to those obtained using the conventional Lang method.

The monochromatic beam topographs were taken at the monochromatic beam station E2, whose optical schema is shown in Fig. 2. The most important part of setup is the monochromator equipped usually with two silicon crystals cut respectively along (111) and (511) planes set in 333 and 511 reflections. The setup enabled the elimination of the most unwanted harmonic radiation. Piezoelectric equipment controlled by feedback with the signal from the ionization chamber provided a necessary stabilization of the monochromator.
In the case of highly deformed, e.g. elastically bent samples, the double-crystal topography is often highly restricted to a narrow stripe. In such situations a very helpful is so-called "zebra pattern" technique [12], when a series of exposures with stepwise altered angular position is recorded on the same film.

Experimental results included in the present paper were obtained from crystals grown in the Institute of Electronic Materials Technology by the Czochralski method. In particular the oxide crystals were grown in the Oxide Crystal Department using the Oxypuller 05-03 equipment made in France by Cyberstar. The inductive heating was used. The charge materials were usually of at least $4 \mathrm{~N}$ grade qualities. The thermal system consisted of high $3 \mathrm{~N}$ grade platinum crucible and passive platinum afterheater. The growing atmosphere usually contained the admixture of oxygen.

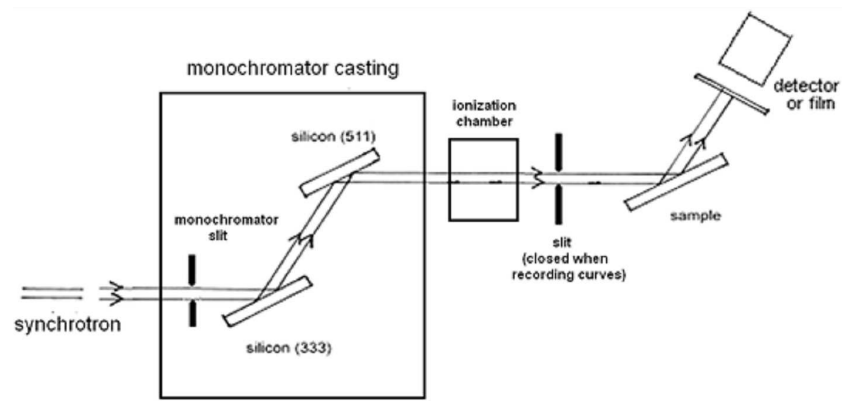

Fig. 2. The scheme of the monochromatic beam arrangement used for taking the topographs and recording of local rocking curves - usually using the radiation of $0.115 \mathrm{~nm}$.

\section{The formation of contrast}

The very important parameter, characterizing the possibilities of revealing the defects is the intrinsic range of reflection predicted by the dynamical theory [25]:

$$
\delta=\frac{2|C|}{\sin \left(2 \vartheta_{b}\right)} \sqrt{\frac{\gamma_{0}}{\left|\gamma_{h}\right|} \chi_{h} \chi_{\bar{h}}}
$$

The polarization factor $C=|\cos 2 \Theta|$ for the $\pi$-polarization in the plane of diffraction and $C=1$, for $\sigma$-polarization perpendicular to the plane of diffraction, $\chi_{h, 0}$ are the coefficient of the Fourier expansion of dielectric susceptibility, proportional to the structural factors $F_{h} \equiv F_{h k l}$

$$
\chi_{h}=-\left(\frac{e}{m c^{2}}\right) \frac{\lambda^{2}}{\pi V} F_{h} .
$$

$V$ is the volume of the elementary cell, $\lambda$ is the radiation wavelength, $e$ and $m$ are the elementary charge and mass of the electron, respectively.

In most often case of "extinction contrast" forming in white beam projection topography in the Bragg case and in the Laue case at small absorption, the additional intensity is contributed by a region of defect "outside the reflection range" fulfilling the condition [55, 25]: 


$$
\left|4 \sin \Theta \frac{\partial \boldsymbol{u}(\boldsymbol{r}) \cdot \boldsymbol{h}}{\partial s_{h}}\right|>\delta,
$$

$\boldsymbol{u}(\boldsymbol{r})$ is the displacement vector connected with the defect, $\boldsymbol{h}$ is the diffraction vector and $s_{h}$ is the coordinate along the reflected beam. The above condition points that wider images of dislocations are obtained in the case when the width of the reflection range is small.

The contrast in transmission topographs is dependent on the absorption - if the attenuation is very strong then the anomalous transmission contrast is observed - and the defects are revealed thanks to the destruction of the so-called "anomalous transmission". The last effect consists in fitting of the excited wave-field to the reflecting planes causing significant decrease of absorption. Due to the anomalous transmission it is otherwise possible to obtain the transmission topographs in highly absorbing crystals built of the element with large atomic number, because the anomalous absorption can be even of the two orders lower than the normal photoelectrical absorption. In addition, the synchrotron sources offer another great help in studying of such crystals thanks to the possibility of using the reflections fulfilling the Bragg condition for very short wavelength and thus with low absorption.

A different contrast mechanism is present in the case of the multicrystal topography, which may be interpreted as the result of local shifts of the rocking curve (with respect to a reference area) caused either by strain field (dislocations) or chemical compositions changes (segregation effects). The observed change of the reflected intensity $\Delta I$ may be written

$$
\Delta I=\frac{\mathrm{d} P(\Theta)}{\mathrm{d} \Theta}\left[\frac{\Delta d}{d} \tan \Theta+\Delta \Theta_{\mathrm{g}}\right] .
$$

The first term describes the change of the Bragg angle and the second the misorientation component in the plane of diffraction. The derivative of rocking curve $P(\Theta)$ is taken at the angular setting where the topograph is exposed. This means that the contrast strongly depends on the angular setting. It is the strongest on the slopes of the rocking curve and inverts coming to the opposite slope. In some cases the topographs taken at the slope allow to reveal the lattice parameter changes $\Delta a / a$ smaller than $10^{-8}$.

The images of individual defects are often accompanied by some interference effects often caused by the decomposition of wave-fields. The obtaining of the theoretical simulated images reproducing such fringes is possible with the use of numerical integration of the Takagi-Taupin equations [25, 24].

\section{Revealing of dislocations and mosaic block structures}

In most of the applications the important criterion for the quality of the crystals is the absence of a mosaic structure as well as the low concentration of dislocations and inclusions, which can negatively affect the properties of crystals important for the application. In particular, in semiconductor crystals a high concentration of dislocation can decrease the carrier mobility and increase the break-down possibility. In case of crystals destined for laser technology the dislocations and block structure can significantly decrease the efficiency of the laser effect.

The synchrotron diffraction topography offers higher possibilities of revealing dislocations than conventional topographic methods exploring $\mathrm{X}$-ray radiation from the sealed X-ray tubes but there are still some limitations concerning the observation of individual dislocations. The most important concerns the dislocation density which should not exceed $5 \times 10^{4} \mathrm{~cm}^{-2}$. The results depend on the specific topographic method and crystal properties, but also on the reflection and the radiation wavelength forming the particular topograph.

The images of dislocations obtained with the use of various synchrotron topographic methods in some Czochralski grown crystal are shown in Fig. 3.

The interesting feature of the topographs reported in Fig. 3a, which is possible in the case of synchrotron topography and availability of very short high energy wavelength, is obtaining of direct (extinction) contrast in case of high absorbing gallium arsenide. Thanks to the narrow rocking curves the images of dislocations in Fig. 3a are quite wide and exhibit double character. The last effect comes directly from the character of the deformation field, and it was explained by Authier [55] in the case of conventional X-ray diffraction topographs.

The images of dislocations outcropping to the surface obtained in the case of the multi-crystal topographs taken on the slopes of the rocking curve maximum have usually the form of the black and white rosettes [8], whose appearance is dependent on the dislocation type. A relatively simple form of the rosette is shown in the simulated image of the screw dislocation reported in Fig. 3d and this image corresponds to the many of dislocation images visible in Fig. 3c. Contrary to the multi-crystal (monochromatic beam) topography, the images of dislocations in the white beam reflection projection topographs (Fig. 3b) have the form of relatively tiny "commas" (or short stripes when the dislocations outcrop the surface at very low angle). It should be noted that reflection topographs in some cases cannot provide sufficient contrast and visibility of dislocation.

The white beam projection topography became very useful in the case when the crystal contains a higher concentration of defects, particularly block structures or some complexes of dislocations such as glide bands and grain boundaries. As examples we reproduce in Figs. 4 and 5 images of $\mathrm{YVO}_{4}$ and $\mathrm{GdVO}_{4}$ crystals with some grain structure. The reproduced topograph reveals a number of irregular white or black stripes depending on the mutual disorientation between blocks. The black stripes correspond to the overlapping of the images from two neighbouring crystal blocks, while the white lines correspond to the gap between the two divergent images. The mutual misorientation of the blocks can also be followed in the section topographs as it is shown in Fig. 5b. 

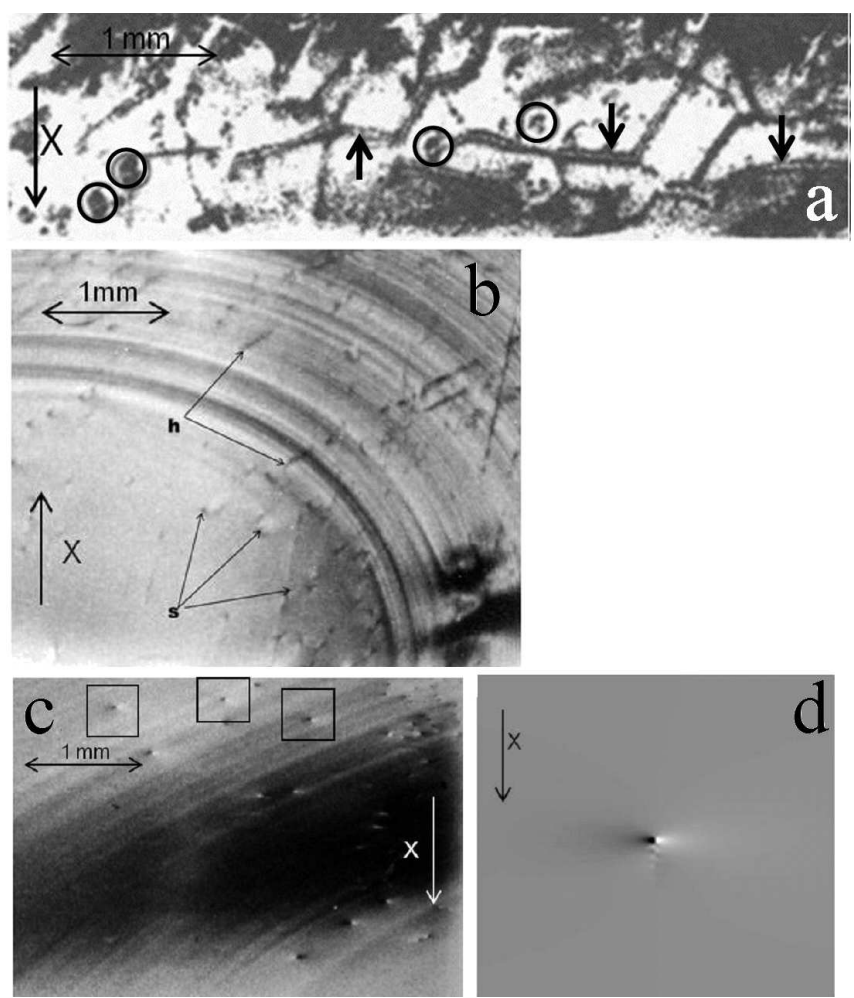

Fig. 3. The synchrotron topographic images of dislocations obtained in Czochralski-grown semiconductor and oxide crystals. (a) Transmission topograph of dislocations in GaAs crystal - according to Wierzchowski et al. [38] - here the arrows denote the dislocations revealed with a double contrast and circles mark the contrasts caused by point-like inclusions. (b) White beam reflection topographic image of a silicon-germanium crystal revealing both dislocation outcropping the surface of the crystal at relatively steep angle (marked s) and low angle (marked h). (c) Monochromatic beam topograph of an yttrium aluminum garnet $\mathrm{Y}_{3} \mathrm{Al}_{5} \mathrm{O}_{12}$ (YAG) crystal substrate wafer. (d) Simulated images of a screw dislocation outcropping the surface in monochromatic-beam topography assuming the parameters for YAG and similar to some images visible in topograph (c) marked representatively with rectangles. The field of the simulation is equal to $1 \mathrm{~mm} \times 1 \mathrm{~mm}$.

The developed block structure being dominating imperfection in $\mathrm{YVO}_{4}$ and $\mathrm{GdVO}_{4}$ single crystals is generally caused by thermal stresses occurring in the process of growth and cooling of the crystals. Additional information about the distribution of the defect in the whole crystal and possible sources of defects may be obtained by following the samples cut out from different places of the crystals and especially of samples cut along the crystal.

White beam synchrotron radiation back-reflection projection topographs enable some evaluation of lattice disorientation between neighbouring blocks. This may be significantly improved by additional exposing of section topographs - topographs recorded using a beam limited by a slit. The image of the slit - due to the disorientation of grains present in the illuminated region of the crystal - is divided into a few mutually displaced segments, in some cases even slightly bent (Fig. 5b). The evaluated disorientation between various blocks was in the range of several arc minutes.

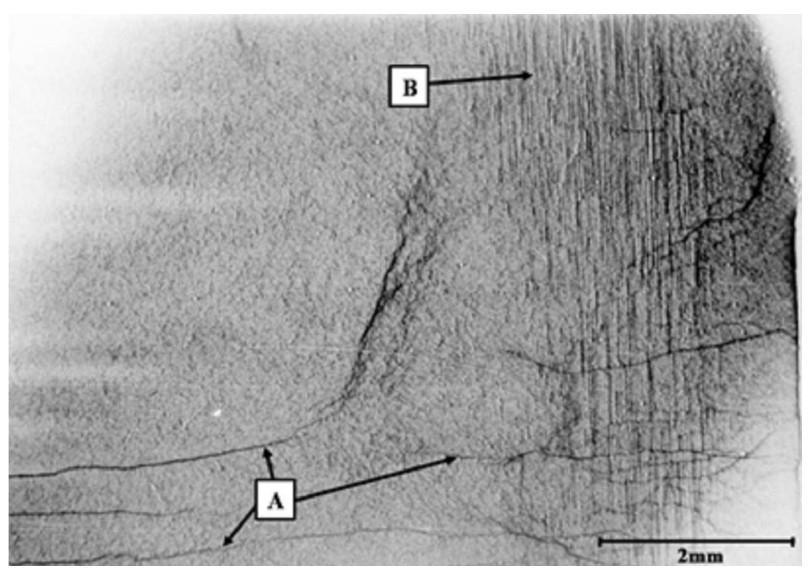

Fig. 4. The synchrotron radiation white beam reflection projection topograph of a sample cut out near the seed of an undoped $\mathrm{YVO}_{4}$ crystal $(d=300 \mu \mathrm{m})$, direction of the beam projection along the normal to (010) planes; A - representative subgrain boundaries, B representative glide bands. According to K. Wieteska et al. [39].

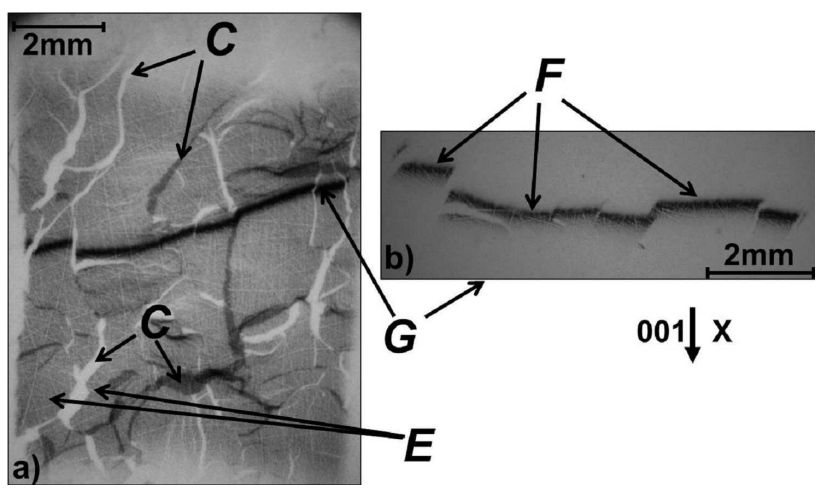

Fig. 5. Synchrotron white beam back-reflection diffraction topographs of $\mathrm{GdVO}_{4}$ crystals: sample cut out from the near end part of the crystal doped with $\operatorname{Er}(0.5$ at.\%); (a) projection topographs with superimposed section topographs (images of the slit); (b) section topographs. $X$ denotes projection of the synchrotron radiation beam direction on the film, $C$ grain boundaries, $E$ - displaced images of misoriented blocks, $G$ - image of the linear slit, $F$ - displaced fragments of the slit image corresponding to the various crystal blocks according to E. Wierzbicka et al. [40].

An interesting example of a slip band structure was revealed in $\langle 001\rangle$ oriented $\mathrm{Ca}_{0.26} \mathrm{Ba}_{0.74} \mathrm{Nb}_{2} \mathrm{O}_{6}$ crystal. In this crystal the glide band structure was formed around the core, and the identification of the contrasts as glide 


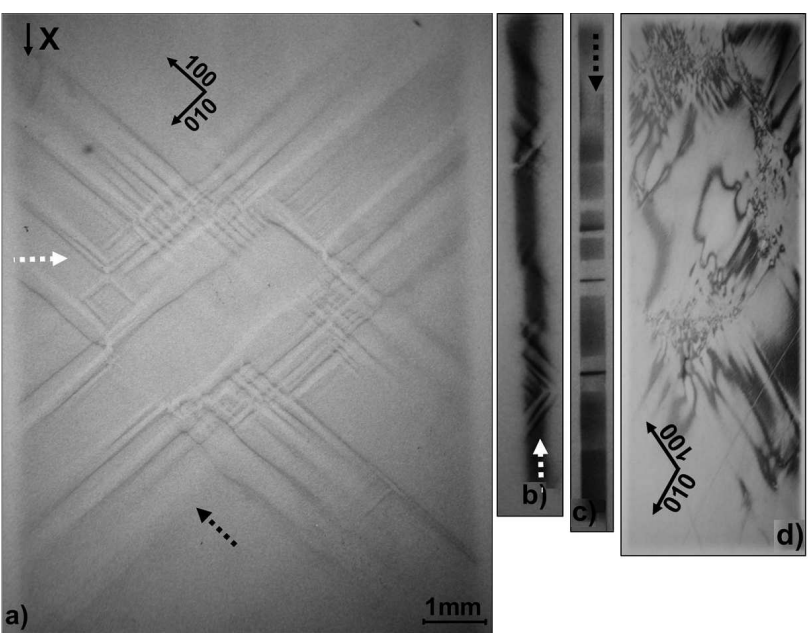

Fig. 6. The synchrotron back-reflection projection topographs of a sample cut out from the end region of $\langle 001\rangle$ oriented $\mathrm{Ca}_{0.26} \mathrm{Ba}_{0.74} \mathrm{Nb}_{2} \mathrm{O}_{6}$ crystal: (a) white beam projection topograph, (b) the back-reflection section topograph taken along the direction marked by white arrow, (c) the transmission section topograph taken along the direction marked by black arrow and (d) the monochromatic beam zebra pattern topograph in the 004 reflection of the $0.1115 \mathrm{~nm}$ wavelength. $\mathrm{X}$ denotes the projection of the incident beam direction on the film - according to K. Wieteska et al. [49].

bands was based on the detailed analysis of the topographs taken in various reflections. In Fig. 6 we show a representative set of topographs including the reflection projection, section (both reflection and transmission) and monochromatic beam zebra pattern topographs. The important observation is the indication of significant misorientations (order of single arc minutes) of crystal fragments separated by dense glide bands - acting as the grain boundary [49].

\section{Revealing of the segregation fringes and defects related to facetted growth}

The grain boundaries are often accompanied by other defects. That is the case of a sample cut out from a $\mathrm{SrLaGaO}_{4}$ crystal (Fig. 7) where some grain structure is visible in the middle region, while the surrounding region contains a dense volume defect being the kind of a cellular growth. It may be noticed that the contrast of the grain boundaries undergoes some contrast inversion in different reflections [41].

The synchrotron investigation can reveal significant strains associated with the segregation fringes [42]. In particular they can be revealed in transmission and reflection section topography, similarly as it was discussed in [47]. The characteristic results were obtained in the case of $\mathrm{Ca}_{0.5} \mathrm{Sr}_{0.5} \mathrm{NdAlO}_{4}$ single crystal grown by the Czochralski method in the [100] direction, which was investigated by means of conventional projection X-ray topography, and by means of synchrotron white beam

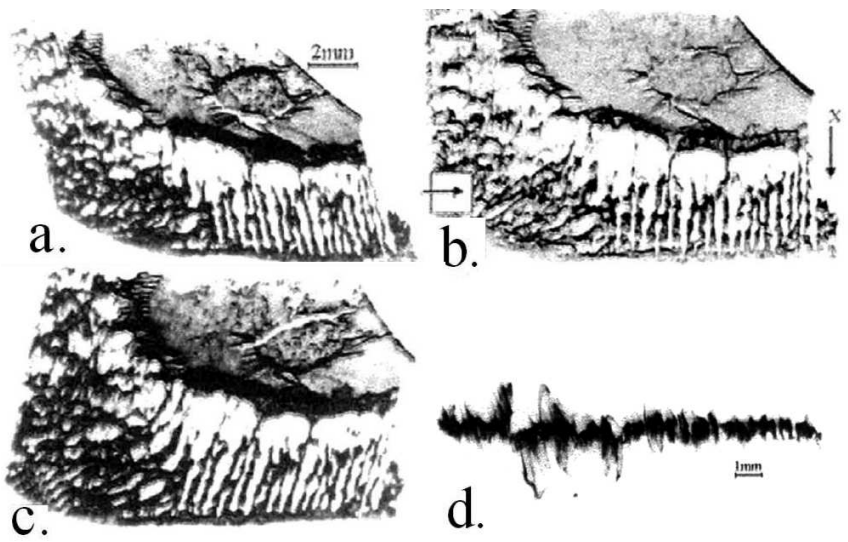

Fig. 7. Synchrotron radiation white beam reflection projection topographs: (a) $h \bar{k} l$, (b) $h 0 l$, (c) $h k l$ reflections, respectively, (d) back reflection section topograph, $(h 0 l)$ reflection; the horizontal arrow in (b) indicates place of taking the topograph. The projection of incoming wave vectors is marked by $\bar{X}$ - according to K. Wieteska et al. [41].

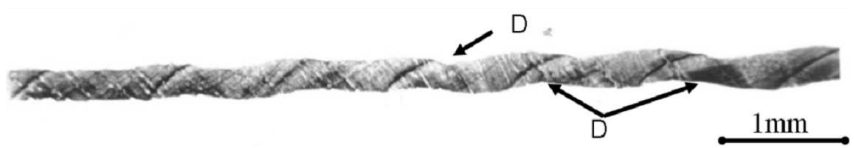

Fig. 8. Synchrotron radiation white beam transmission section topograph in $\overline{3} 6 \overline{7}$ reflection for the sample cut from $\mathrm{Ca}_{0.5} \mathrm{Sr}_{0.5} \mathrm{NdAlO}_{4}$ single crystal, grown by the Czochralski method, $\lambda=0.047 \mathrm{~nm}, \mu t=2.3$ ( $\mu$ - is the linear absorption coefficient, $t$ is the sample thickness); D denotes characteristic kinks of the section topograph which may indicate the deformation of the lattice according to M. Malinowska et al. [42].

and monochromatic beam topography [42]. The crystal seems to be very interesting in view of very high lattice parameter changes and strains connected with the composition changes and the presence of large amount of volume defects interpreted as the solute trails. The topographs are shown in Figs. 8-10.

A significantly strong effect of lattice deformation associated with striation was revealed in particular by white beam transmission section topographs, shown in Fig. 8. One can notice characteristic kinks of the section topographs which may indicate the deformation of the lattice (marked by D). It should be noted that the white beam topographs can reveal only the change of lattice orientation, which may be evaluated taking the ratio of the kink height to the distance of the particular spot (topograph) from the crystal. The simple evaluation gives the values on the level of $0.001^{\circ}$.

Long-range lattice deformation of the sample was also revealed by the monochromatic beam topographs shown in Fig. 9. Figure 9a presents the topograph taken at the maximum of the local rocking curve recorded with $50 \mu \mathrm{m} \times 50 \mu \mathrm{m}$ probe beam in the middle region of the topograph. It shows narrow stripes of complicated shapes, 


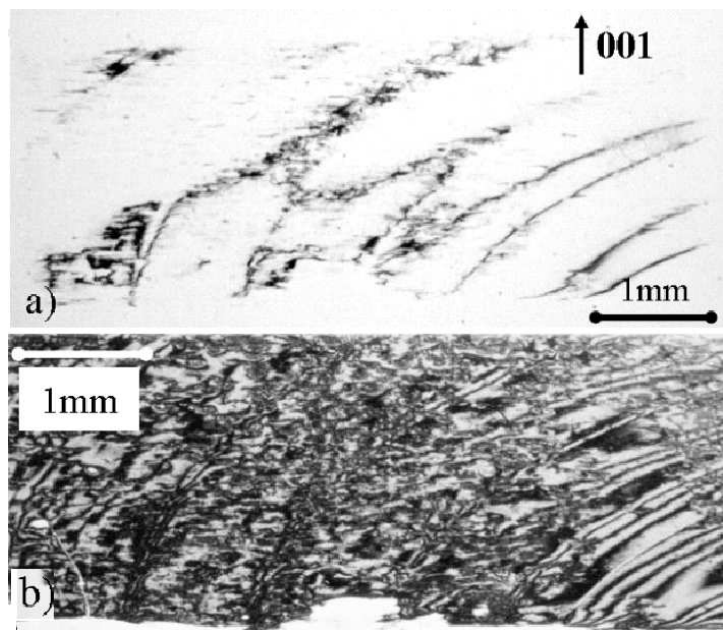

Fig. 9. Synchrotron radiation monochromatic back reflection projection topographs, 400 reflection, $\lambda=$ $0.111 \mathrm{~nm}$; (a) topograph at the maximum of the rocking curve, (b) "zebra pattern" - superimposed images taken at different angular position of the crystal $\left(0.003^{\circ}\right.$ apart). According to M. Malinowska et al. [42].

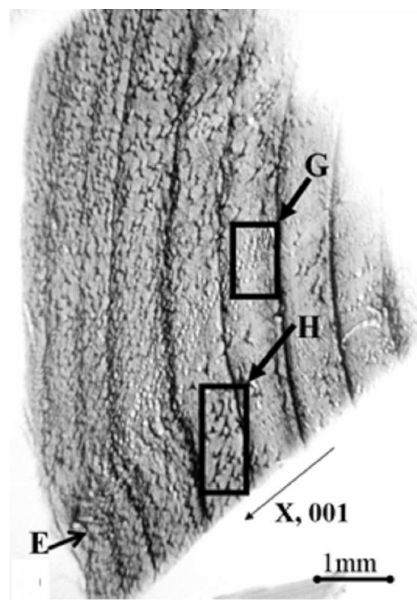

Fig. 10. Synchrotron radiation white beam back reflection projection topograph in $13 \overline{4}$ reflection, $\lambda=$ $0.094 \mathrm{~nm}$; E denotes boundary between two slightly misoriented regions and $\mathrm{G}, \mathrm{H}$ - defect groups with two kinds of diffraction contrasts. According to A. Malinowska et al. [42].

partly related with growth striations. The "zebra pattern" (Fig. 9b) obtained by superposition of several images (as in Fig. 6b) taken at different angular crystal positions $\left(0.003^{\circ}\right.$ apart $)$ covers the whole area $8 \mathrm{~mm} \times 3.4 \mathrm{~mm}$ illuminated by the beam.

Apart of the striations, the topographs reveal a considerable amount of resolved individual contrasts. The diffraction contrasts in the form of short black and white lines are seen between the striations.

Some further details of resolved defects were revealed by the synchrotron white beam back reflection projection topographs of the sample shown in Fig. 10 when the projection of the incident beam is parallel to the [010] direction. The topographs reveal a relatively high density $\left(\approx 10^{3} \mathrm{~cm}^{-2}\right)$ of defects forming at least two kinds of contrasts. The first category of defects (marked by G in Fig. 10) form round bright diffraction contrasts with some dark parts on their boundaries. It suggests that the contrast is associated with precipitates - possibly the solute trails. The second category forms "bird" shaped strong dark contrasts (marked by $\mathrm{H}$ in Fig. 10). In the last case all of them form almost the same contrast in particular topographs with the same directions of the "wings". The interpretation of the defects belonging to the first category, as the kind of rod-like volume defects, was confirmed by the numerical simulation of the topographic image shown in Fig. 11.

The present simulations of white beam back reflection projection topographs were obtained by adding many shifted spherical images, filling the area irradiated by the beam. The application of the model enabled obtaining realistic simulation of the monochromatic beam topographic images of some solute trails in garnets and the Bragg-case section images of some defects resembling rod-like inclusions described in [56]. The approximation of the strain field of rod-like defects was obtained by a summation of the contributions from the point-like inclusions used in the formulae derived from those given by Sen [57].

Another example of the simulations confirming the expected presence of rod-like inclusions in case of SLG crystals is presented in Fig. 12. The simulation corresponds to assumed shrinkage type inclusion at the angular setting for low angle flank maximum and large angle flank, respectively.

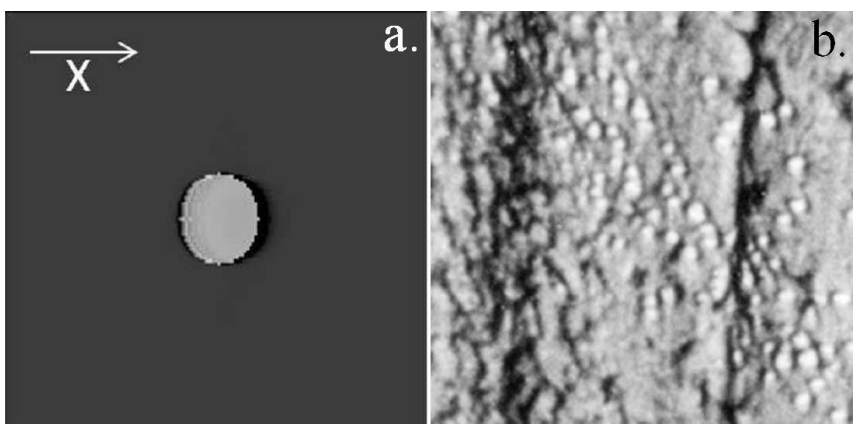

Fig. 11. (a) Simulated projection topograph of the rod-like inclusion, compared with enlarged fragment of the white beam projection topograph of $\mathrm{Ca}_{0.5} \mathrm{Sr}_{0.5} \mathrm{NdAlO}_{4}$ (b) with the volume defects which may be attributed to rod-like defects. The field of simulation is $1 \mathrm{~mm} \times 1 \mathrm{~mm}$ (according to [58]).

In Fig. 13 we present the white beam topograph of $\mathrm{Yb}_{3} \mathrm{Al}_{5} \mathrm{O}_{12}$ (YbAG) crystal doped with $1.5 \%$ erbium revealing segregation fringes and sets of facets [50]. The schematic positions of identified facets are shown in Fig. 13b. The facets are formed, when the actual growth surface coincides with low indiced crystallographic plane, which usually grows slowly. For identification of the 

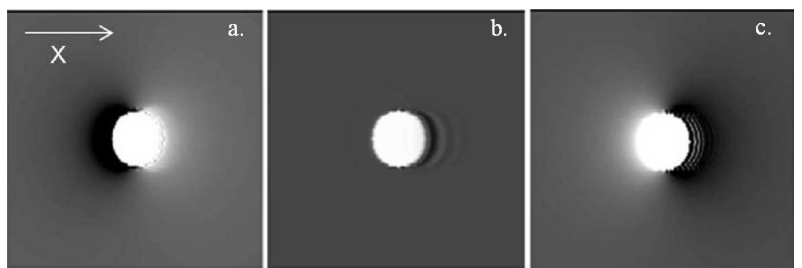

$1 \mathrm{~mm}$

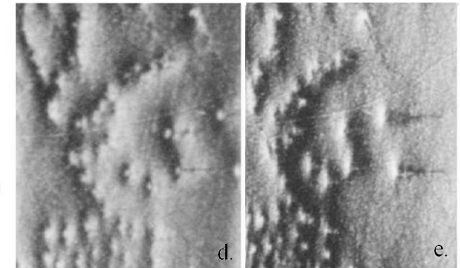

Fig. 12. $(\mathrm{a}-\mathrm{c})$ Simulated monochromatic beam images of shrinking rod-like inclusions of $150 \mu \mathrm{m}$ in diameter for angular setting respectively at low angle flank, maximum and high angle flank of the rocking curve. (d), (e) The monochromatic images of volume inclusions in SLG crystals exhibiting some features of rod-like inclusions respectively for low and high angle flank of the rocking curve. The contrast of the defects is reversed for the two flanks (according to [58]).

facets it was essential to determine the shape of the growth surface and it was possible by exposing of transmission section topographs with the beam perpendicular to the sample reported in Fig. 14. These patterns revealed the section of the growth surface of regular spherical shape whose curvature was easy evaluable knowing the actual thickness of the sample.

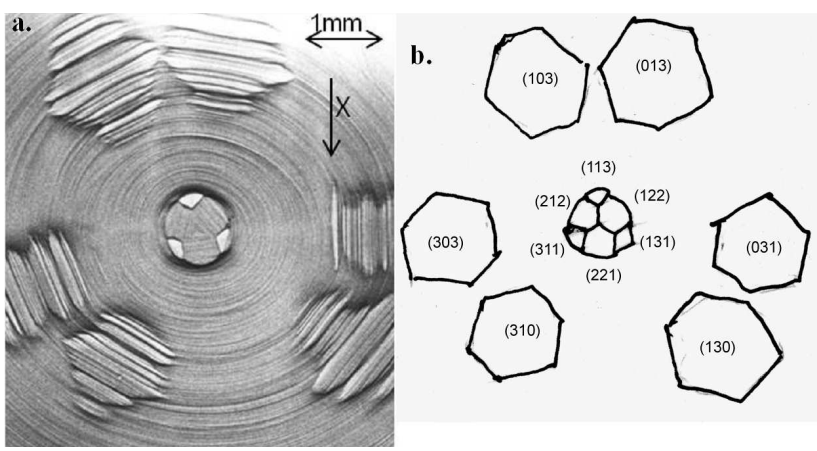

Fig. 13. (a) White beam projection back reflection topograph of $\mathrm{Yb}_{3} \mathrm{Al}_{5} \mathrm{O}_{12}$ crystal doped with $1.5 \%$ erbium revealing segregation fringes and sets of facets and (b) schematic position of the identified facets - according to K. Kołodziejak et al. [50].

The important results were obtained in case of diffraction topographic investigations of CSBN crystals with the composition obeying the formula $\left(\mathrm{Ca}_{0.28} \mathrm{Ba}_{0.72}\right)_{x}\left(\mathrm{Sr}_{0.61} \mathrm{Ba}_{0.39}\right)_{1-x} \mathrm{Nb}_{2} \mathrm{O}_{6}$ with $x=0.25$, 0.50 , and 0.75 . Contrary to previously studied SBN and CBN crystals we did not observe any glide bands in the CSBN. The most characteristic defects were segregation fringes and some rod-like inclusions around the

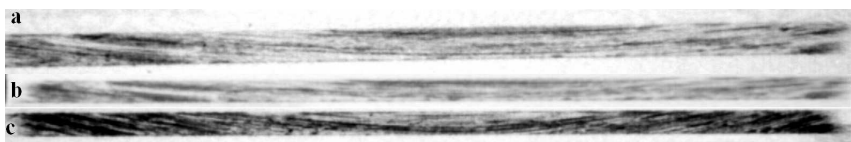

Fig. 14. Transmission white beam section topographs of $\mathrm{Yb}_{3} \mathrm{Al}_{5} \mathrm{O}_{12}$ sample revealing the striation fringes corresponding to the successive location of growth surfaces in the plane intersected by the narrow incident beam perpendicular to the sample. The topogaphs allow for the evaluation of the inclination of growth surfaces necessary for identification of the facets: (a) crystal with $10 \%$ erbium, (b) crystal with $30 \%$ erbium, (c) crystal with $1.5 \%$ erbium - according to K. Kołodziejak et al. [50].

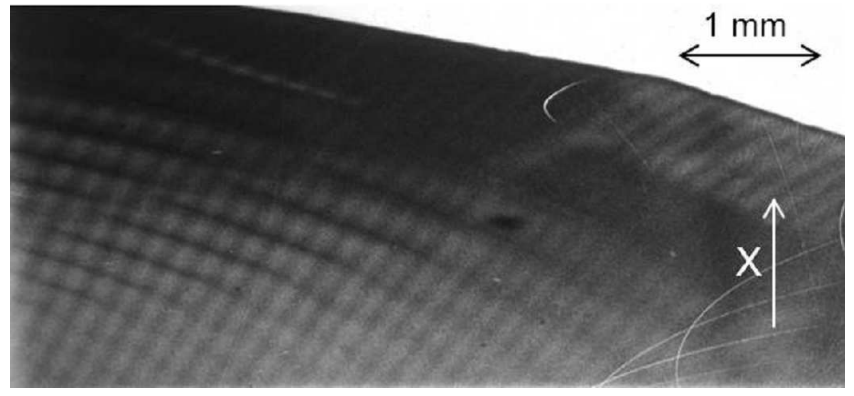

Fig. 15. Monochromatic beam topography of the near edge part of CSBN crystal where two systems of segregation form a characteristic crossing pattern. $\mathrm{X}$ denotes the projection of the incident beam direction (according to [59]).

core present in the central part of samples cut from different crystals. In addition, the topographs revealed a very characteristic phenomenon. This consisted in the presence of two different systems of segregation fringes forming the crossing pattern as it may be seen in the two reproduced monochromatic beam topographs of the sample cut-out from crystal with $x=0.75$ (Fig. 15). The visibility of these both systems of segregation was much better in the monochromatic beam topographs than in the white beam ones. We also observed the inversion of contrast for two different slopes of the rocking curve maximum. As it may be noticed, one of the systems is connected with the subsequent position of the growth surface being coaxial with the core and the boundaries of the crystal. The second system crossing the previous one cannot be connected with the position of growth surface. In addition, by taking the topographs from both sides of relatively thick crystal wafer we confirmed that this fringe system is relatively slow changing, hence it propagates through relatively large distances along the growth axis. The possible explanation of the observed phenomenon is the kind of ghost pattern reproducing some chemical composition changes in the new part of the growing crystal.

\section{Observation of the twin domain structure}

The synchrotron topography can be used for revealing of the domain structure. The representative illustration 


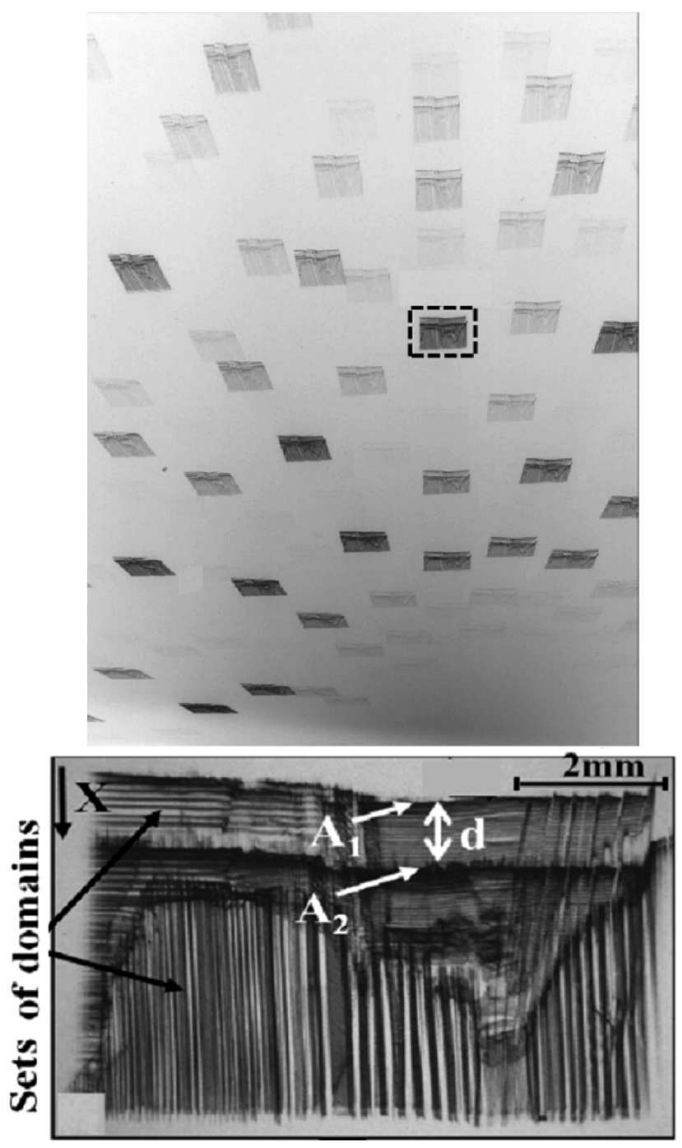

Fig. 16. Top - example of full white beam synchrotron pattern of $\mathrm{LaAlO}_{3}: \operatorname{Pr}(4.2 \%$ at.), $\mathrm{Mg}(2.1 \%$ at.) crystal confirming the twin character of the domains. One can notice that the images of all different domains are present in the vicinity of each Laue spot - the marked region corresponds to the white beam projection topography corresponding to mixed 620 and 310 pseudo-cubic reflection shown on the bottom and revealing several sets of the misorientation domains according to K. Wieteska et al. [45]. The projection of the diffraction vector is located vertically.

of the crystal containing twinning domains is shown in Fig. 16. It has been confirmed that the domains are of a twin character, similarly as it was described by Dudley and Yao in $\mathrm{LaAlO}_{3}$ and $\mathrm{LaGaO}_{3}$ crystals [36, 37]. In those crystals the twin configuration and their changes were studied as a function of temperature in the vicinity of phase transition temperatures.

The domain systems, observed by us, were investigated in $\operatorname{Pr}_{x} \mathrm{La}_{1-x} \mathrm{AlO}_{3}$ (for different $x$ ) and in $\mathrm{LaAlO}_{3}$ crystals doped with $\mathrm{Pr}$, and their appearance and configuration were compared for samples, containing different amount of praseodymium. The observed twin domains systems $[45,46]$ were located perpendicular to $\langle 100\rangle$ and $\langle 110\rangle$ (pseudo-cubic) directions. This means that they should be located in the $\{110\}_{\text {rhom }}$ and $\{211\}_{\text {rhom }}$ planes, which correspond to $\{100\}_{\text {pcub }}$ and $\{110\}_{\text {pcub planes in }}$ the pseudocubic lattice, respectively, as it was suggested by Dudley and Yao [37].

\section{Conclusions}

The diffraction topography became very popular in view of its nondestructive character and the possibilities of the interpretation of defects in terms of crystallography and theory of elasticity. Similarly as in the case of other X-ray methods the significant development of X-ray diffraction topography was caused by the availability of the synchrotron radiation, which offered some important advantages. These are in particular the possibilities of choosing the most suitable wavelength, achieving spatial resolution under $1 \mu \mathrm{m}$ and, more effective, of collimation of the beam and very high intensities, enabling shortening of the time necessary for the investigation.

The paper provided an opportunity to present the possibilities of synchrotron diffraction topography for studying the Czochralski grown crystals. In fact the crystals grown with this method are the majority of applications of both conventional and synchrotron diffraction topography. It was possible to illustrate these possibilities of synchrotron topography with a number of results obtained in some years of investigations at HASYLAB by the present authors.

\section{Acknowledgments}

The important part of synchrotron investigations were supported by the HASYLAB projects II 20100001 EC and I 20110423 EC.

\section{References}

[1] W.F. Berg, Naturwissenschaften 19, 391 (1931).

[2] C.S. Barrett, Trans. AIME 161, 391 (1945).

[3] G.N. Ramachandran, Proc. Indian Acad. Sci. A 19, 280 (1944).

[4] A. Guinier, J. Tennevin, Acta Crystallogr. 2, 133 (1949).

[5] W.L. Bond, J. Andrus, Am. Mineralogist 37, 37 (1952).

[6] L.G. Schultz, Trans. AIME 200, 1082 (1954).

[7] A.R. Lang, Acta Metall. 5, 358 (1957).

[8] A.R. Lang, J. Appl. Phys. 29, 597 (1958).

[9] B.K. Tanner, X-Ray Diffraction topography, Pergamon Press, Oxford 1976.

[10] U. Bonse, E. Kappler, Z. Naturforsch. 214, 16 (1958).

[11] U. Bonse, Z. Phys. 153, 278 (1958).

[12] M. Renninger, Adv. X-ray Anal. 10, 32 (1967).

[13] M. Renninger, Z. Naturforsch. A 19, 783 (1964).

[14] T. Tuomi, K. Naukkarinen, P. Rabe, Phys. Status Solidi (a) 25, 93 (1974).

[15] M. Hart, J. Appl. Crystallogr. 8, 436 (1975).

[16] B.K. Tanner, M. Safa, D. Midgley, J. Bordas, J. Magn. Magn. Mater. 1, 337 (1976).

[17] B.K. Tanner, Prog. Cryst. Growth Character. 1, 23 (1976). 
[18] M. Hart, M. Sauvage, D.P. Siddons, Acta Crystallogr. A 36, 947 (1980).

[19] J.F. Petroff, M. Sauvage, J. Cryst. Growth 43, 628 (1978).

[20] M. Sauvage, Nucl. Instrum. Methods 152, 313 (1978).

[21] R.W. Whatmore, P.A. Goddard, B.K. Tanner, Nature 299, 44 (1982).

[22] H. Cerva, W. Graeff, Phys. Status Solidi (a) 87, 507 (1985).

[23] W. Graeff, K. Wieteska, J. Sci. Technol. 3, 162 (1992).

[24] D.K. Bowen, B.K. Tanner, High Resolution X-ray Diffractometry and topography, Taylor and Francis, London 1998.

[25] A. Authier, Dynamical Theory of X-Ray Diffraction, Oxford University Press Inc., New York 2001.

[26] K. Wieteska, Acta Phys. Pol. A 86, 545 (1994).

[27] M. Moore, Crystallogr. Rev. 18, 207 (2012).

[28] A.R. Lang, A.P.W. Makepeace, M. Moore, W.K. Wierzchowski, C.M. Welbouurn, Philos. Trans. R. Soc. Lond. A 337, 497 (1991).

[29] J. Baruchel, M. DiMichiel, T. Lafford, P. Lhuissier, J. Meyssonier, H. Nguyen-Thi, A. Philip, P. Pernot, L. Salvo, M. Scheel, C.R. Phys. 14, 208 (2013).

[30] A. Malinowska, M. Lefeld-Sosnowska, J. Hartwig, J. Appl. Crystallogr. 46, 48 (2013).

[31] E. Kasper, N. Burle, S. Escoubas, J. Werner, M. Oeheme, K. Lyutovich, J. Appl. Phys. 111, 063507 (2012).

[32] S. Yao, X. Hu, T. Yan, H. Liu, J. Wang, X. Qin, Y. Chen, J. Appl. Crystallogr. 43, 276 (2010).

[33] M. Muehlberg, M. Burianek, B. Joschko, D. Klimm, A. Danilewsky, M. Gelissen, L. Bayarjargal, G.P. Görler, B.O. Hildmann, J. Cryst. Growth 310, 2288 (2008).

[34] I.A. Prokhorov, B.G. Zakharov, A.S. Senchekov, A.V. Egorov, D. Camel, P. Tison, J. Cryst. Growth 310, 4701 (2008).

[35] Y. Yoneda, J. Mizuki, H. Takeda, T. Shiosaki, IEEE Trans. Ultrason. Ferroelectr. Freq. Control 55, 971 (2008).

[36] G.-D. Yao, S.Y. Hou, M. Dudley, J.M. Phillips, J. Mater. Res. 7, 1847 (1992).

[37] M. Dudley, G.-D. Yao, J. Phys. D 26, A120 (1993).

[38] W. Wierzchowski, K. Wieteska, W. Graeff, Acta Phys. Pol. A 91, 1015 (1997).

[39] K. Wieteska, W. Wierzchowski, E. Wierzbicka, A. Malinowska, M. Lefeld-Sosnowska, T. Łukasiewicz, W. Graeff, Acta Phys. Pol. A 114, 438 (2008).

[40] E. Wierzbicka, A. Malinowska, K. Wieteska, W. Wierzchowski, M. Lefeld-Sosnowska, M. Świrkowicz, T. Łukasiewicz, C. Paulmann, Acta Phys. Pol. A 121, 906 (2012).

[41] K. Wieteska, W. Wierzchowski, W. Graeff, M. LefeldSosnowska, A. Pajaczkowska, E. Wierzbicka, A. Malinowska, J. Alloys Comp. 401, 75 (2005).
[42] A. Malinowska, M. Lefeld-Sosnowska, K. Wieteska, W. Wierzchowski, W. Graeff, A. Pajaczkowska, J. Cryst. Growth 310, 3398 (2008).

[43] K. Wieteska, W. Wierzchowski, W. Graeff, M. LefeldSosnowska, M. Regulska, Acta Phys. Pol. A 101, 729 (2002).

[44] M. Lefeld-Sosnowska, E. Olszyńska, W. Wierzchowski, K. Wieteska, W. Graeff, A. Pajączkowska, A. Kłos, J. Alloys Comp. 382, 153 (2004).

[45] K. Wieteska, W. Wierzchowski, A. Malinowska, S. Turczyński, M. Lefeld-Sosnowska, D.A. Pawlak, T. Łukasiewicz, C. Paulmann, Acta Phys. Pol. A 121, 910 (2012).

[46] K. Wieteska, W. Wierzchowski, A. Malinowska, S. Turczyński, M. Lefeld-Sosnowska, D.A. Pawlak, T. Łukasiewicz, W. Graeff, Acta Phys. Pol. A 117, 268 (2010).

[47] K. Wieteska, W. Wierzchowski, W. Graeff, M. LefeldSosnowska, M. Regulska, J. Phys. D 36, A133 (2003).

[48] K. Wieteska, W. Wierzchowski, W. Graeff, M. LefeldSosnowska, M. Regulska, Mater. Sci. Eng. B 91-92, 462 (2002).

[49] K. Wieteska, W. Wierzchowski, A. Malinowska, M. Lefeld-Sosnowska, M. Swirkowicz, T. Łukasiewicz, C. Paulmann, Radiat. Phys. Chem. in press (2013).

[50] K. Kołodziejak, W. Wierzchowski, K. Wieteska, M. Malinowski, W. Graeff, T. Łukasiewicz, Cryst Res. Technol. 43, 369 (2008).

[51] W. Wierzchowski, K. Wieteska, W. Graeff, H. Sakowska, T. Łukasiewicz, M. Pawłowska, Cryst. Res. Technol. 40, 517 (2005).

[52] D. Savytskii, A. Senyshyn, A. Matkowskii, L. Vasylechko, K. Wieteska, W. Wierzchowski, T. Łukasiewicz, U. Bismayer, Z. Kristallogr. 218 17 (2003).

[53] W. Wierzchowski, K. Wieteska, W. Graeff, M. Pawłowska, B. Surma, S. Strzelecka, J. Alloys Comp. 362, 301 (2004).

[54] A. Malinowska, M. Lefeld-Sosnowska, K. Wieteska, W. Wierzchowski, J. Härtwig, W. Graeff, Phys. Status Solidi (a) 206, 1816 (2009).

[55] A. Authier, Adv. X-Ray Anal. 10, 9 (1967).

[56] T. Balcer, W. Wierzchowski, K. Wieteska, Acta Phys. Pol. A 117, 333 (2010).

[57] R. Sen, J. Quant. Mech. VIII, 365 (1949).

[58] W. Wierzchowski, T. Balcer, K. Wieteska, A. Malinowska, M. Lefeld-Sosnowska, K. Mazur, Experimental and Numerically Simulated Bragg-Case Topographic Images of Rod-Like Inclusions, Hasylab Annual Report, 2010.

[59] K. Wieteska, W. Wierzchowski, A. Malinowska, M. Lefeld-Sosnowska, M. Swirkowicz, T. Łukasiewicz, M. Romaniec, E. Wierzbicka, C. Paulmann, Observation of Ghost Segregation Pattern in CSBN Crystals, Hasylab Annual Report, 2012 